\title{
The dilemma of refractory epileptic syndromes without structural lesions visible through conventional MRI. "Sight beyond sight": is it possible to trust what we cannot see?
}

\author{
O dilema das síndromes epilépticas refratárias sem lesões estruturais visíveis através da \\ RM convencional. "Visão além do alcance”: é possível confiar no que não podemos ver?
}

Antônio José da Rocha

Seção de Neurorradiologia, Santa Casa de Misericórdia de São Paulo, Sao Paulo SP, Brazil.

\section{Correspondence:}

Antônio José da Rocha

Santa Casa de Misericórdia de São

Paulo / Serviço de Diagnóstico por

Imagem

Rua Dr. Cesário Motta Junior 112,

Vila Buarque

01221-020 São Paulo SP - Brasil

E-mail:antonio.rocha@grupofleury.

com.br

Conflict of interest:

There is no conflict of interest to declare.

Received 11 November 2013 Accepted 18 November 2013

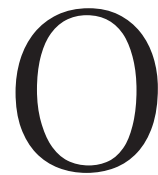

ver the last few decades, several different modern techniques of nonconventional magnetic resonance imaging (MRI) have provided clinicians with new perspectives on both old diseases and prior clinical issues. Refractory epilepsies are an old clinical issue that reflect those conditions for which therapies employing two or more antiepileptic drugs (AEDs), separately or in association, have been unable to control the epilepsy. The mechanisms of drug resistance are multifaceted, and they are likely to be variable and multifactorial, but at least in part attributed to the underlying cause of epilepsy ${ }^{1}$. It is assumed that patients with drug-resistant epilepsy have increased risks of premature death, injuries, psychosocial dysfunction, and a reduced quality of life ${ }^{1}$.

Appropriate MRI protocols remain essential for studying patients with epilepsy and to identify the majority of those patients who manifest structural lesions. Nevertheless, in a number of patients with epilepsy, MRIs are unremarkable, particularly if there is a disorder of cortical development, typically, a focal cortical dysplasia².

In this issue of Arquivos de Neuro-Psiquiatria, Baldissin and colleagues ${ }^{3}$ advocate the use of texture analysis for MRI and discuss how it may be useful for detecting microstructural abnormalities that may cause refractory epilepsies, by comparing a small set of patients to a matched control group. Their article aims to add new information to this field of research and reinforces the concept that neurological diseases and brain abnormalities may exist even when they are be undetected through conventional MRI.

A variety of neuroimaging modalities have proved to be useful when making the diagnosis of epilepsy and its structural basis ${ }^{1}$. However, several other techniques have also been used to study patients not exhibiting brain abnormalities through conventional MRI, including modern techniques using 3 Tesla magnetic resonance (MR) equipment ${ }^{4}$. A number of different techniques have shown promising results for studying epilepsy, including magnetic resonance spectroscopy (MRS) and diffusion tensor imaging (DTI $)^{5,6}$. All of these techniques have limitations, mainly regarding the detection of neocortical epilepsy without a strong suspicion about the location of the epileptogenic focus or lesion in $\mathrm{MRI}^{2}$. Consequently, attempts to extrapolate the results drawn from well-controlled research to heterogeneous clinical practice have been weak.

Texture analysis for MR images is a quantitative method used to mathematically study variations in grey-level tones from different selected brain regions using manually selected areas as well as to use computational models to automatically detect dysplastic lesions $^{7-9}$. In fact, these techniques have emerged in the context of refractory epileptic syndromes as promising tools for detecting in vivo subtle abnormalities in the temporal lobe and extra-temporal areas that are invisible to human visual inspection ${ }^{7-9}$. However, external factors, including MR parameters, the acquisition process, and the occurrence of 
artifacts, may disturb the final results for both manual and automatic techniques. Additionally, the manually selected brain areas may also interfere with the reproducibility of such techniques.

As the authors have stressed, the current article has several limitations, including its small sample of patients and the absence of a correlation with pathology. The absence of a gold-standard parameter and the poor clinical and EEG correlation reduce the impact of the conclusions drawn by the researchers. In clinical settings, video-EEG and MRI results should be invariably inspected to improve imaging interpretation and to correlate structural and functional abnormalities.
More recently, when video-EEG and MRI results have been found to be inconclusive, 18F-fluorodeoxyglucose-positron emission tomography (18-FDG-PET) has been promoted as an extremely helpful tool.

Despite the efforts of many researchers, there is still an opportunity to a new facet of the not-uncommon clinical and radiological dilemma of refractory epileptic syndromes accompanied by unremarkable conventional MRI findings. I hope that texture analysis for MRI can be incorporated into the currently available armamentarium, supplanting more expensive techniques, with the aims of improving imaging diagnoses and benefitting larger samples of patients.

\section{References:}

1. Kwan P, Schachter SC, Brodie MJ. Drug-resistant epilepsy. N Engl J Med 2011;365:919-926.

2. Cendes F. Neuroimaging in investigation of patients with epilepsy. Continuum 2013;19:623-642.

3. Baldissin MM, Souza EM. Investigation of magnetic resonance imaging texture analysis as an aid tool for characterization of refractory epilepsies. Arq Neuropsiquiatr 2013;71:920-924.

4. Zijlmans M, de Kort GA, Witkamp TD, et al. 3T versus 1.5T phasedarray MRI in the presurgical work-up of patients with partial epilepsy of uncertain focus. JMRI 2009;30:256-262.

5. Willmann O, Wennberg R, May T, Woermann FG, Pohlmann-Eden B. The role of $1 \mathrm{H}$ magnetic resonance spectroscopy in pre-operative evaluation for epilepsy surgery. A meta-analysis. Epilep Res 2006;71:149-158.
6. Winston GP, Yogarajah M, Symms MR, McEvoy AW, Micallef C, Duncan JS. Diffusion tensor imaging tractography to visualize the relationship of the optic radiation to epileptogenic lesions prior to neurosurgery. Epilepsia 2011;52:1430-1438.

7. Castellano G, Bonilha L, Li LM, Cendes F. Texture analysis of medical images. Clin Radiol 2004;59:1061-1069.

8. Bonilha L, Kobayashi E, Castellano G, et al. Texture analysis of hippocampal sclerosis. Epilepsia 2003;44:1546-1550.

9. Antel SB, Collins DL, Bernasconi N, et al. Automated detection of focal cortical dysplasia lesions using computational models of their MRI characteristics and texture analysis. Neurolmage 2003;19:1748-1759. 